\title{
An Experimental Study on the Manufacturing Properties of Solid Capsules for Mixing Cementitous Composite Materials for Self- Healing of Crack
}

\author{
Yun-Wang Choi ${ }^{1}$, Sung-Rok Oh ${ }^{1 *}$, and Byung-Keol Choi ${ }^{1}$ \\ ${ }^{1}$ Department of Civil Engineering, Semyung University, 2713665 Semyung-ro, Jecheon-si, \\ Chungcheongbuk-do, South Korea
}

\begin{abstract}
In this study, we fabricated self-healing solid capsules using cement powder, an inorganic-based material that is the same as that of cement composites. Self-healing solid capsules can be divided into two stages(capsule core preparation and capsule wall coating). First, the capsule core mixes the core material and the coagulant to form a core material dough having a proper quality, and then puts into a capsule core manufacturing machine. the next, it was produced through the pore chamber under the pressure of the capsule core making machine. Second, The capsule wall is formed by placing the prepared capsule core into the chamber of the capsule wall coating apparatus and then applying the wall material while the chamber is rotating. The prepared capsules were mixed with cement composites to evaluate fluidity and strength. That is, we evaluated the basic performance changes of cement composite materials with capsules. We will use the results of this paper as the base data to consider the optimal amount of capsule at the level that satisfies the performance required when mixing capsules.
\end{abstract}

\section{Introduction}

Smart structures and intelligent materials have emerged as new trends for reducing cracks in concrete, and extensive research is being performed on the self-healing of concrete cracks. Structures capable of self-healing are able to detect cracks in the early stage and heal themselves, thereby saving time and costs involved in maintenance and repair compared to general structures. A major advantage of self-healing is that repairs can be easily carried out, even for structures that are difficult to access physically.[1,2]

Among the various methods of enabling self-healing, one approach is to encapsulate materials that have self-healing capabilities and introduce them as capsules. Because such capsules can hold a large amount of self-healing materials [3, 4], reactions can be selectively induced at cracks. In one international study, self-healing materials and catalysts were encapsulated in the form of micro-sized capsules[5, 6]. Meanwhile, in Korea, self-healing

\footnotetext{
*Corresponding author: cgdbs02@nate.com
} 
micro-sized capsules capable of reacting with sunlight or moisture without catalysts have been applied in actual construction[7]. Two conclusions can be derived from these case studies at home and abroad: First, the target of self-healing capsules is not the structure's body, but its surface. Second, An organic material is used as the self-healing material.[8, 9].

The number of capsules for self-healing is limited as they are normally utilized as coating to protect the surface, thus making it difficult to heal deep cracks. most of the structure's body is comprised of inorganic composite materials. therefore, the self-healing performance can be enhanced by using inorganic materials that have the same properties instead of organic materials that have different thermal properties. While direct mixing the cementitious composite materials, the thickness of the matrix can be freely adjusted as necessary. The self-healing performance can also be maximized by introducing more selfhealing capsules.

This study developed solid capsules that can be directly mixed with cementitious composite materials using inorganic capsules with the same properties as that of cementitious composite materials. The properties of the self-healing solid capsules were also evaluated. This study did not consider the healing effect of self-healing capsules. Instead, it focused on evaluating the effects of capsule mixing on the quality of cementitious composite materials. The results of this study can serve as a valuable reference for developing self-healing capsules and capsule-containing cementitious composite materials.

\section{Experiment outline}

\subsection{Fabrication of self-healing solid capsules (SHSC)}

\subsubsection{Core material}

Self-healing solid capsules (SHSC), which are inorganic self-healing capsules, were fabricated from core materials comprised of cement powder. The cement used in the experiments was ordinary Portland cement. Generally, cement has a mechanism to react by moisture.[10] Therefore, in order to granulate the cement powder, a coagulant having a low water content should be used.

The coagulant used in this study was High-purity methyl alcohol, which facilitates coagulation and is easy to evaporate, was used as the coagulant. The coagulated cement powder was inserted into the capsule fabricating equipment in tempered form to create the core material. Left of Fig. 1 shows a capsule core manufacturing apparatus.

Table 1. Chemical components and physical properties of cement.

\begin{tabular}{|c|c|c|c|c|c|c|c|c|c|}
\hline $\begin{array}{c}\text { Items } \\
\text { Types }\end{array}$ & $\begin{array}{c}\mathbf{C a O} \\
(\%)\end{array}$ & $\begin{array}{c}\mathrm{SiO}_{2} \\
(\%)\end{array}$ & $\begin{array}{c}\mathrm{Al}_{2} \mathbf{O}_{3} \\
\mathbf{( \% )}\end{array}$ & $\begin{array}{c}\mathrm{MgO} \\
(\%)\end{array}$ & $\begin{array}{c}\mathbf{F e}_{2} \mathbf{O}_{3} \\
\mathbf{( \% )}\end{array}$ & $\begin{array}{c}\mathbf{S O}_{3} \\
\mathbf{( \% )}\end{array}$ & $\begin{array}{c}\text { L.O.I } \\
(\%)\end{array}$ & $\begin{array}{c}\text { Surface } \\
\text { area } \\
\left(\mathbf{c m}^{2} / \mathbf{g}\right)\end{array}$ & $\begin{array}{c}\text { Density } \\
\left(\mathbf{g} / \mathbf{c m}^{3}\right)\end{array}$ \\
\hline OPC & 61.40 & 21.60 & 3.40 & 2.50 & 3.10 & 2.50 & 0.03 & 3,540 & 3.15 \\
\hline
\end{tabular}




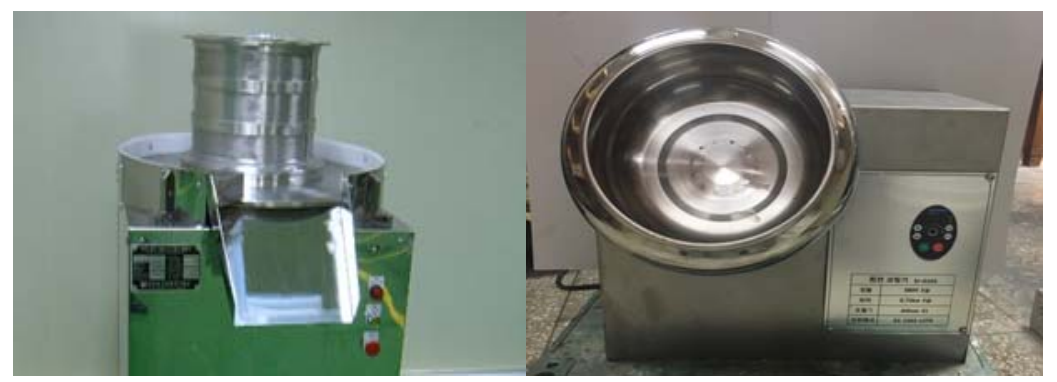

Fig. 1 Manufacture of capsules (capsule core fabricating equipment ; left, Wall material coating equipment ; right)

\subsubsection{Wall material}

The core material, in which cement is coagulated, can react reactions with the mixing water during the mixing with cementitious composite materials. also it can lost due to the effects of cement composites and mixers. Accordingly, two types of wall materials have been selected to ensure moisture barrier and strength.

The wall materials were liquid rubber (Liquid type Rubber, LR) and an acryl-based material (Poly(methyl methacrylate), PMMA). Methylene chloride $\left(\mathrm{CH}_{2} \mathrm{Cl}_{2}, \mathrm{MC}\right)$ was used as a dissolving agent to melt the solid PMMA.

The performance of each wall material was assessed by applying the test methods specified in KS L 5105 after standard mortar mixing. Moisture resistance was assessed through the permeability test specified in KS F 4930, and strength was evaluated for samples at day 3 according to KS L 5105. The fabricated core material was inserted into the chamber, and the wall material was lubricated and dried while the chamber rotated at a certain RPM. Right of fig. 1 (b) shows a wall material coating equipment apparatus.

\subsection{Quality of SHSC}

\subsubsection{Slump flow}

The slump flow of cementitious composite materials according to mixing of SHSC was assessed using paste samples, fabricated by excluding fine aggregates from the plain mix specified under KS L 5105. SHSC selected 4 levels(0, 5, 10, and 15 mass $\%)$ for cement mass.

\subsubsection{Compressive strength}

The compressive strength of cementitious composite materials according to mixing of SHSC was assessed using paste samples, fabricated by excluding fine aggregates from the plain mix specified under KS L 5105. 


\section{EXPERIMENTAL RESULTS AND DISCUSSION}

\subsection{Fabrication of SHSC}

\subsubsection{Consistency}

SHSC is produced by preparing capsule core and coating the wall. If the tempering state is too thin or thick, it is difficult to fabricate the core material. This study utilized OPC as the core material, and applied methyl alcohol at three levels (Step-1: 10, 20, 30 mass\%) of the OPC mass to obtain the desired consistency. For quantitative analysis of consistency, a table flow test was performed, and consistency standards were established based on the slump values.

Fig. 2 give the shape of Step-1 samples immediately after tempering and the results of the table flow test. The results show that samples of $10 \%$ and $20 \%$ consistency were easily coagulated, but the table flow test could not be carried out, as the samples were crushed even by the slightest shock. The $30 \%$ samples were too watery and obtained a flow, not a table flow. The core material fabricator creates a core material at a consistency suitable for the chamber, and coagulates the material using pressure created by the chamber lid and mixing blades within the chamber. Therefore samples of $10 \%$ and $20 \%$, which are unable to withstand shock, are not suitable since they will be pulverized when the wall material is applied or during drying. Samples of $30 \%$ cannot coagulate if they are too watery after the core material is formed. As such, an adequate consistency can be achieved between $20 \%$ and $30 \%$. To obtain a suitable consistency, the experiments were repeated with the coagulant at five mixing ratios (Step-2: 21, 22, 23, 24, and 25 mass \%).

Fig. 3 show the shape of Step-1 immediately after tempering and the results of the table flow test. Samples of $21 \%$ coagulated immediately after mixing, but measurements were difficult due to the exterior collapsing. Samples of $22 \%$ and $23 \%$ maintained the coagulated form, and slump values were obtained based on the table flow results. Similar to the results for $30 \%$ samples, samples of $24 \%$ and $25 \%$ were too watery to be coagulated, and the flow values were insignificant. These results indicate that an adequate consistency in creating the core material can be achieved by mixing the coagulant at $22-23 \%$.

Fig. 4 show the production and loss of the core material after mixing the coagulant at $22 \%$ and $23 \%$. Samples of $22 \%$ had a $5 \%$ loss in mass, while samples of $23 \%$ showed segregation of the coagulant due to pressure within the chamber because of their consistency relatively watery. The latter also had a greater loss in mass with the capsule cores sticking to one another. The production efficiency of the capsule core can be enhanced by mixing the coagulant at $22 \pm 0.5 \%$ when creating the core material. 


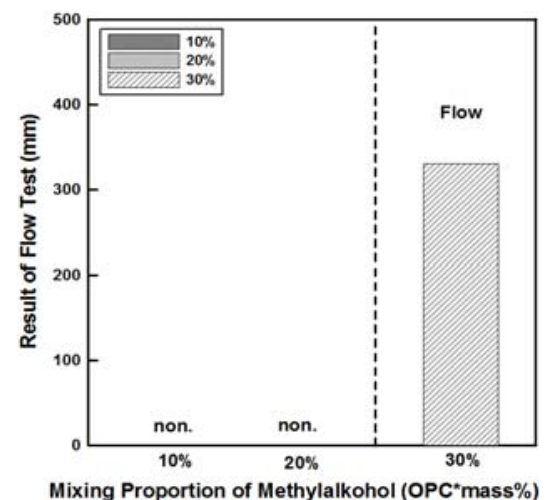

Fig. 2 Result of flow test on group of step-1

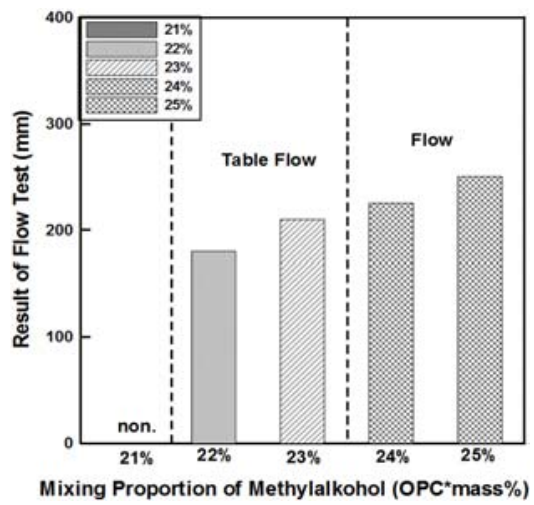

Fig. 3 Result of flow test on group of step-2

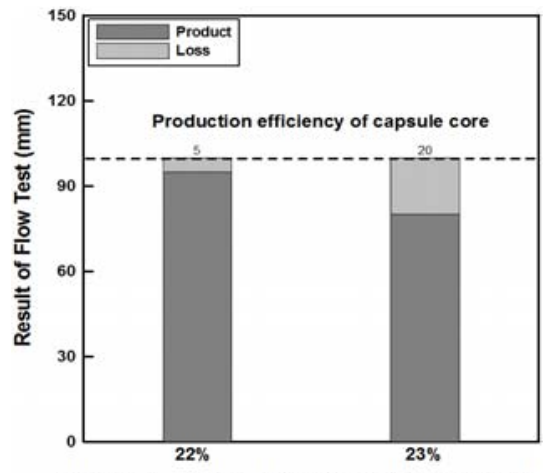

Mixing Proportion of Coagulant (OPC ${ }^{\star}$ mass $\%$ )

Fig. 4 Production efficiency of capsule core 


\subsubsection{Properties of wall materials}

Since the capsule core has a mechanism that reacts with moisture, moisture must be blocked before the material is destroyed by cracks. so, that it can be possesses activation energy for self-healing. Therefore, the choice of wall material is important. To assess the properties of the wall material, permeability and compressive strength tests were performed after standard mortar mixing.

Fig. 5 and Fig. 6 show the results of the permeability and compressive strength tests. The permeation amounts of LR and PMMA were smaller than the plain sample without any wall material by $88 \%$ and $57 \%$, respectively. Compared to PMMA, the permeation amount of LR decreased by $74 \%$. This can be attributed to PMMA being diluted 20 times in the dissolvent, and the less uniform coating of the dissolvent due to the higher viscosity of PMMA than LR. Even if the dissolvent is uniformly coated, the remaining PMMA can form a coating layer after the dissolvent evaporates, resulting in a change in effective content.

Fig. 6 shows that the compressive strength of LR and PMMA is higher than that of the plain sample by approximately $15.8 \%$ and $12.7 \%$, respectively. The effects of PMMA dissolution on effective content and coating require further review . However, in this study, LR was selected as the wall material and applied to the capsule core.

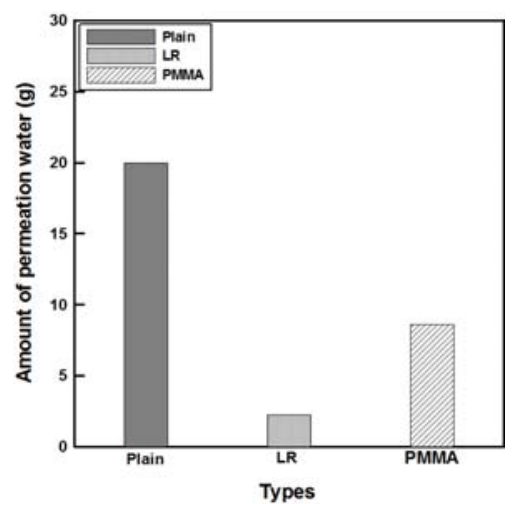

Fig. 5 Amount of permeation water according to wall material

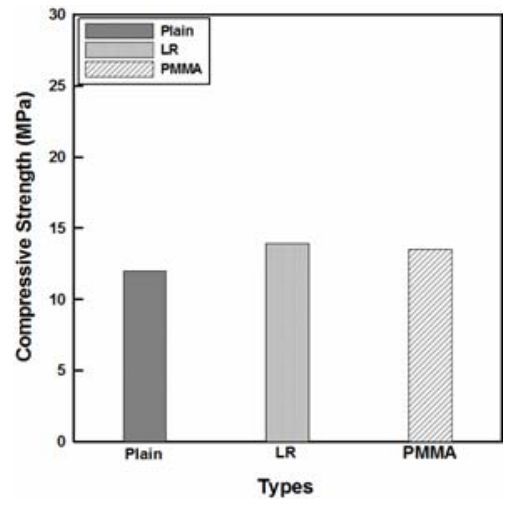

Fig. 6 Compressive strength according to wall material 


\subsubsection{The shape and size of SHSC}

Fig. 7 shows the shape and size of the SHSC. The SHSC is in the form of a spherical shape, and it has a size of more than about 1,000 micrometers. The coating of the wall material was assessed through a qualitative evaluation.

Left of fig. 8 shows a case where the wall material is not coated, and right of Fig. 8 shows a state after the wall material is coated. In the case of the former, since the wall material is not coated, when the capsule core is supplied with water, it is immediately absorbed. In the latter case, since the wall material is coated, even if water is supplied to the capsule core, it is not absorbed.

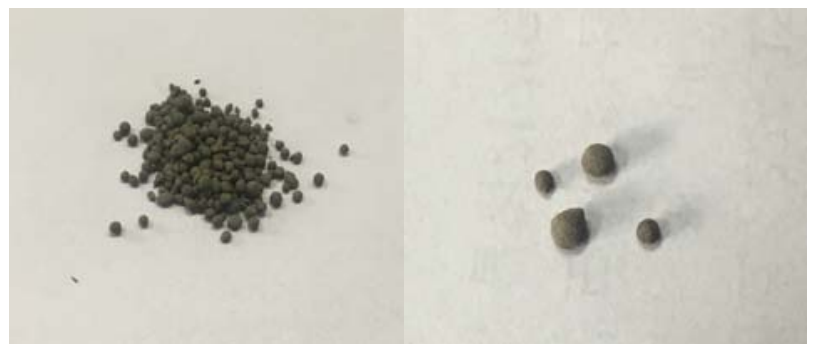

Fig. 7 The shape and size of SHSC

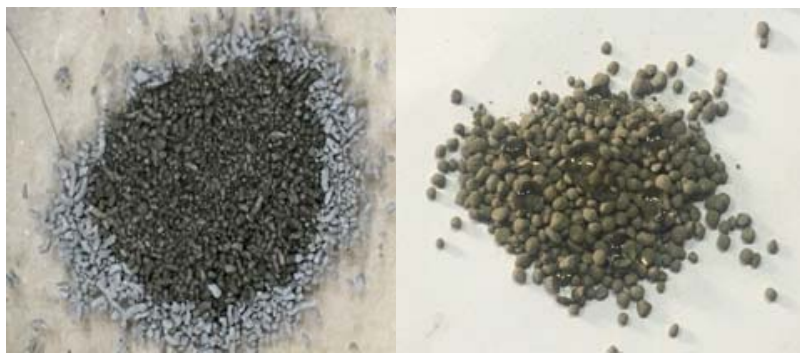

Fig. 8 Qualitative evaluation of wall matarial coating (before coating ; left, after coating ; right)

\subsection{Quality of SHSC}

\subsubsection{Loss rate}

Fig. 9 shows the result of SHSC loss evaluation. The SHSC loss was assessed by mixing the cement composites immediately after mixing, and then washing was carried out in a sieve. And it was dried. As can be seen from Fig. 9, the loss was less than 5\% for the paste after mixing, and about 10 to $15 \%$ for the mortar. When SHSC are mixed with the paste and mortar, they are presumed to be influenced by the mixing blades during mixing. Since mixing with the mortar involves fine aggregates, the rate of loss increases by two to three times compared to the paste. This loss can be compensated by reinforcing the wall material. 


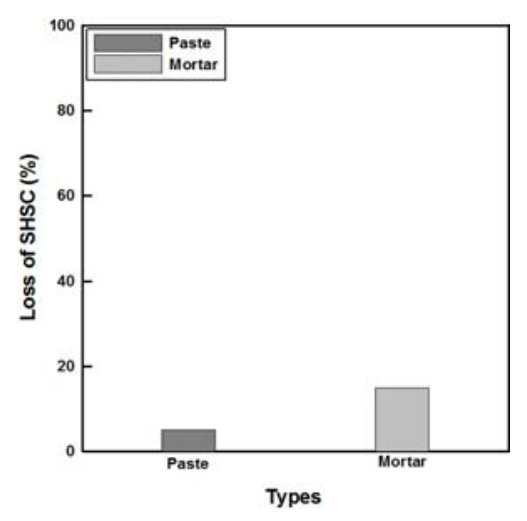

Fig. 9 Loss of SHSC immediately after mixing with cementitious composites

\subsubsection{Slump flow}

Fig. 10 show the slump flow in relation to the mixing ratio of SHSC. Compared to the plain samples without mixing, the slump flow decreased by $5 \%$ with a $5 \%$ increase in the mixing ratio. It is considered that SHSC affects the fluidity of cement composites. Further study is needed to examine the possibility of the decrease in slump flow arising from reactions with the mixing water in the case of the wall material having a non-uniform coating.

On the other hand, if the coating is uniform, and assuming that there is no influence on moisture, the SHSC grains are presumed to have caused the decrease in fluidity. The decrease in fluidity with an increase in SHSC, which are needed for the self-healing performance of cementitious composite materials, can be compensated by using binders other than cement or chemical admixtures. Another issue to be considered, although falling outside the scope of this study, is the distribution of SHSC in the cementitious composite materials.

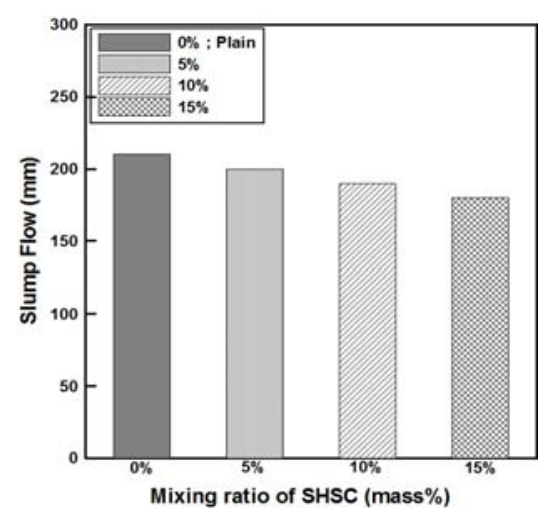

Fig. 10 Slump flow according to mixing ratio of SHSC

\subsubsection{Compressive strength}

Fig. 11 shows the compressive strength in relation to the mixing ratio of SHSC. Compared to the plain samples without mixing SHSC, the compressive strength decreases 
proportionately with a $5 \%$ increase in the mixing ratio of SHSC. Because the cement powder in coagulated form is distributed in the matrix of cementitious composite materials, the areas in which SHSC are present are relatively weaker, resulting in a smaller compressive strength. Since the weak areas are cross-sections containing SHSC, hydration reactions may be induced at cracks with the leaking of SHSC through cracks, thereby enabling the self-healing of cracked sections.

However, the cracked part can not be healed by the SHSC alone on the entire cracked area., but a significant portion may be restored by adding a function to facilitate swelling or expansion. Considering how strength decreases by at least $15 \%$ at a $5 \%$ mixing ratio of SHSC, the strength of cementitious composite materials can be improved by adjusting the material proportions, adding additional binders, or using chemical admixtures. The adequate mixing ratio of SHSC should be examined with consideration of the efficiency of compressive strength in relation to self-healing performance.

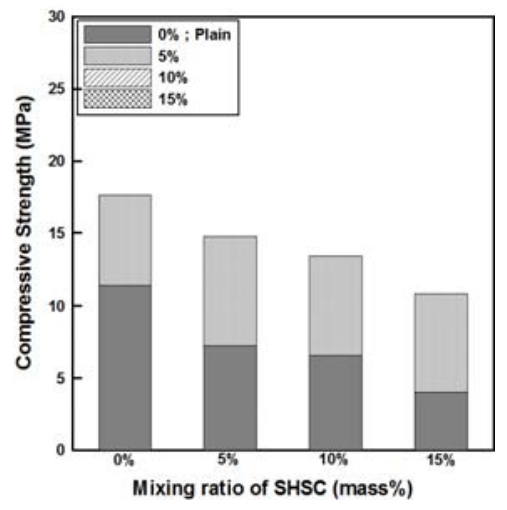

Fig. 11 Compreessive Strength according to mixing ratio of SHSC

\section{CONCLUSION}

This study developed solid capsules that can be directly mixed with cementitious composite materials using inorganic capsules with the same properties as that of cementitious composite materials. The properties of the self-healing solid capsules were also evaluated. This study focused on evaluating the effects of capsule mixing on the quality of cementitious composite materials. The following conclusions were derived:

1. When cement powder was utilized as the core material, the optimal coagulant mixing ratio for adequate consistency could be obtained. However, the tests have to be repeated to obtain the optimal coagulant mixing ratio if the core material includes a material other than cement powder or consists of at least two materials.

2. Capsule core coating was performed with liquid rubber as the wall material to improve resistance to moisture and strength. However, quantitative analysis on the uniform coating of the wall material is needed, and materials other than liquid rubber must be evaluated.

3. When capsules are mixed with cementitious composite materials, fluidity and compressive strength tend to decrease. It is considered that the quality of the cementitious composites can be improved by adjusting the mixing ratio and improving the chemical mixture to improve the slump and compressive strength.

This research was supported by a grant(17SCIP-B103706-03) from Construction Technology Research Program funded by Ministry of Land, Infrastructure and Transport of Korean government 


\section{References}

[1] Erik Schlangen and Senot Sangadji, Journal of Procedia Engineering, The 2nd International Conference 54, 39-57 (2013)

[2] An, E., J. and Shin, M., S., Proceeding of Korea Concrete Institute 26, No. 2, 477-479 (2014)

[3] Tatyana Nesterova, Kim Dam-Johansena, Journal of Organic Coatings 75, No. 4, 309318 (2012)

[4] M. Hunger, A.G. Entrop, I. Mandilaras, H.J.H. Brouwers and M. Founti, Journal of Cement \& Concrete Composites 31, No. 10, 731-743 (2009)

[5] Shazim Ali Memon, H.Z. Cui, Hang Zhang and Feng Xing, Journal of Applied Energy 139, 43-55 (2014)

[6] Kim, P. S., Jo, C. K., Ju, T. H., Choi, Y. C., and Lee, J. K., proceeding of The Korean Society for Energy, 225-228 (2003)

[7] Song, Y. K., Jo, Y. H., Lim, Y. J., Cho, S. Y., Yu, H. C., Ryu, B. C., Lee, S. I., and Chung C. M., Journal of ACS Applied Materials and Interfaces 5, No. 4, 1378-1384 (2013) [8] Xianfeng Wang, Feng Xing, Ming Zhang, Ningxu Han and Zhiwei Qian, Journal of Materials 6, No. 9, 4064-4081 (2003)

[9] B.J. Blaiszika, N.R. Sottos and S.R. White, Journal of Composites Science and Technology 68, No. 3, 978-986 (2007)

[10] Korea Concrete Institute, Concrete Standard Specification (Korea Concrete Institute, 2009) 